\title{
Large bipartite Bell violations with dichotomic measurements
}

\author{
C. Palazuelos ${ }^{1}$ and Z. Yin ${ }^{2,3, *}$ \\ ${ }^{1}$ Instituto de Ciencias Matemáticas, Universidad Complutense de Madrid, Madrid, Spain \\ ${ }^{2}$ School of Mathematics and Statistics, Wuhan University, Wuhan, China \\ ${ }^{3}$ Institute of Theoretical Physics and Astrophysics, University of Gdańsk, Gdańsk, Poland \\ (Received 4 May 2015; revised manuscript received 9 July 2015; published 10 November 2015)
}

\begin{abstract}
In this paper, we introduce a simple and natural bipartite Bell scenario by considering the correlations between two parties defined by general measurements in one party and dichotomic ones in the other. We show that unbounded Bell violations can be obtained in this context. Since such violations cannot occur when both parties use dichotomic measurements, so far our setting can be considered as the simplest one where this phenomenon can be observed. Our example is essentially optimal in terms of the outputs and the Hilbert space dimension.
\end{abstract}

DOI: 10.1103/PhysRevA.92.052313

PACS number(s): 03.67.Mn, 03.65.Ud

\section{INTRODUCTION}

The famous paper by Einstein, Podolsky, and Rosen [1] doubted the completeness of quantum theory and predicted that there should be a complete theory to explain nature that fulfilled locality and realism. We say that such a theory is classical or that it admits a local hidden variable model (LHVM). Whether nature can be explained by a LHVM became a philosophical rather than a physical debate for a long time. However, in his groundbreaking paper [2], Bell provided an inequality that must be satisfied by all classical probability distributions obtained in a certain measurement setting while at the same time it is violated by some probabilities obtained from quantum measurements. This violation reveals that nonlocality (violation of local realism) is an intrinsic property of quantum theory, which plays an important role in quantum information science.

Ever since Bell's work, a lot of Bell-type inequalities have been studied extensively (see [3] for a review). The significance of studying violations of Bell inequalities is to quantify the deviation of classical and quantum theory, but it is also important in many other aspects of quantum information theory, such as entanglement and Hilbert dimension witness [4-6] and quantum communication complexity [7]. In recent years, violations of Bell inequalities have become more popular because of their crucial role in device-independent quantum cryptography [8-10] and the generation of random numbers $[11,12]$. It is natural that large violations of Bell inequalities provide more benefit, both in theory and applications. In fact, the study of the asymptotic behavior of Bell violations is essential to understand this phenomenon, and the results along these lines have been very useful to learn about certain properties of quantum nonlocality and its relation with other resources [13-16].

Unfortunately, obtaining large Bell violations is a highly nontrivial problem. In particular, when one considers the simplest possible scenario, that is, two observers using dichotomic measurement on a bipartite system as in [2], the "amount of Bell violation" is upper bounded by a constant $1.676<K_{G}<$ 1.783 , as was proved by Tsirelson [17]. In this case, we usually use expectation values to describe the correlations between the two parties, which is equivalent to using the joint probabilities

\footnotetext{
*hustyinzhi@163.com
}

without the marginals. The corresponding inequalities are called correlation Bell inequalities. The limitation proved by Tsirelson leads to a study of two different generalizations: multipartite correlation Bell inequalities and general bipartite Bell inequalities. Recent results have shown that in both contexts there exist unbounded Bell violations.

The research on unbounded violations of Bell inequalities has followed two parallel lines. One is based on operator space theory, which has been developing prosperously in recent years as a branch of functional analysis. Through this approach, in [18] the authors showed that unbounded Bell violations exist for tripartite correlation Bell inequality. This answered in the negative a question posed by Tsirelson about the possibility of a similar result to the one existing in the bipartite case. In the general bipartite case, several works have shown that one can also obtain unbounded Bell violations, and they have also studied how far these results are from being optimal $[15,19]$. On the other hand, strong techniques from computer science have also been used in this problem. Along these lines, in [20-22] the authors established and improved some of the previous results in terms of nonlocal games.

The use of general measurements to obtain large bipartite Bell violations makes the problem difficult to handle, and it entails additional difficulties for experimental realizations. Hence, it is natural to consider a simpler bipartite scenario in which one of the observers uses general measurements while the second one uses dichotomic ones. More precisely, suppose Alice can choose among $N$ different measurement settings labeled by $x=1, \ldots, N$. Each of them can result in one of $K$ outcomes, labeled by $a=1, \ldots, K$. Suppose also that Bob can choose among $N^{\prime}$ different measurement settings labeled by $y=1, \ldots, N^{\prime}$, but each of them has only binary outcomes, labeled by $b= \pm 1$. Then, if we denote by $P=$ $[P(a, b \mid x, y)]_{x, y}^{a, b}$ the associated probability distributions, we can define the following correlations between Alice and Bob:

$$
E(a \mid x, y)=P(a, 1 \mid x, y)-P(a,-1 \mid x, y) .
$$

Such correlations can be viewed as a hybrid of those correlations in general and dichotomic scenarios, since our scenario is a mixture of them.

If the probability distribution $P=[P(a, b \mid x, y)]_{x, y}^{a, b}$ admits a LHVM, then

$$
E(a \mid x, y)=\int_{\Omega} P(a \mid x, \lambda) E(y, \lambda) d \mathbb{P}(\lambda)
$$


for every $a, x, y$. Here, $(\Omega, \mathbb{P})$ is a probability space and $[P(a \mid x, \lambda)]_{a}$ is a probability distribution for all $x, \lambda$. Moreover, $-1 \leqslant E(y, \lambda) \leqslant 1$ for every $y, \lambda$. We will denote the set of all LHV correlations by $\mathcal{L}$.

If $P$ has a quantum realization, then

$$
E(a \mid x, y)=\operatorname{tr}\left(E_{x}^{a} \otimes F_{y} \rho\right)
$$

for every $a, x, y$. Here, $\rho \in B\left(H_{A} \otimes H_{B}\right)$ is a quantum state, $\left(E_{x}^{a}\right)_{x}^{a} \subset B\left(H_{A}\right)$ are POVMs on Alice's part, i.e., $E_{x}^{a} \geqslant 0$, and $\sum_{a \in A} E_{x}^{a}=1$ and $\left(F_{y}\right)_{y} \subset B\left(H_{B}\right)$ are dichotomic observables on Bob's part, i.e., $-\mathbb{1} \leqslant F_{y} \leqslant \mathbb{1}$ for every $y$. We denote the set of all quantum correlations by $\mathcal{Q}$.

Following the standard definitions, for given natural numbers $N, N^{\prime}, K$, we define an asymmetric Bell functional (inequality) $M$ as a set $\left(M_{x, y}^{a}\right)_{x, y}^{a}$ of real numbers with $x=1, \ldots, N, y=1, \ldots, N^{\prime}$, and $a=1, \ldots, K$. For a given correlation $E=[E(a \mid x, y)]_{x, y}^{a}$, we define

$$
\langle M, E\rangle=\sum_{x=1}^{N} \sum_{y=1}^{N^{\prime}} \sum_{a=1}^{K} M_{x, y}^{a} E(a \mid x, y) .
$$

We define the classical and quantum bounds of $M$, respectively, by

$$
\begin{aligned}
& B_{\mathcal{C}}=\sup \{|\langle M, E\rangle|: E \in \mathcal{L}\}, \\
& B_{\mathcal{Q}}=\sup \{|\langle M, E\rangle|: E \in \mathcal{Q}\} .
\end{aligned}
$$

Finally, the quantum violation of $M$ is defined by

$$
L V(M)=\frac{B_{\mathcal{Q}}(M)}{B_{\mathcal{C}}(M)}
$$

The aim of this work is to study whether one can get unbounded violations in this scenario, since it is the simplest bipartite context in which such violations can occur. Here, we will prove that there are indeed Bell inequalities as in (1) leading to unbounded violations. More precisely, we will show in the next section that for every $n$ there exists a Bell inequality $M$ with $N, N^{\prime}=2^{n}$ and $K=n$ such that $L V(M)=$ $\Omega\left(\sqrt{n} / \log ^{2} n\right)$. Even though this example is still far from experimental realizations, the use of dichotomic measurements in Bob's party entails an important simplification with respect to the previous known examples of large violations. In addition, to obtain the previous order, Alice and Bob can share the maximally entangled state and Alice can perform some simple von Neumann measurements on her party. Our approach is based on a modification of the Khot-Vishnoi (KV) game [23] and the results in [21] to obtain an asymmetric version of it, which will work in our setting. Moreover, our result is near optimal (up to a logarithm factor) in terms of Alice's outputs and the Hilbert space dimension (see Propositions 1 and 2). Although the motivation of our approach comes from Banach space theory, the results presented in this paper do not require any knowledge about it. In fact, we will present our results in terms of nonlocal games, where everything becomes very natural.

A nonlocal game $G=G(\pi, V)$ is defined as follows [24]: $\pi$ is a probability distribution on $X \times Y$, and $V$ : $X \times Y \times A \times B \rightarrow\{0,1\}$ is the predicate function, where $X, Y, A, B$ are nonempty finite sets. The referee will randomly choose questions $(x, y) \in X \times Y$ according to $\pi$ and send the questions to two spatially separated players, Alice and Bob. The players (without communication) will answer the questions by $(a, b) \in A \times B$. They win the game if and only if $V(a, b \mid x, y)=1$. Before the game starts, Alice and Bob may agree on some strategy (classical or quantum) to play the game. The classical value of a game $G$ is the maximal winning probability, which is restricted by only using classical strategies. Thus, we have

$$
\omega_{c}(G)=\max _{a, b} \sum_{x, y} \pi(x, y) V(a(x), b(y) \mid x, y),
$$

where the maximum is taken over all functions $a: X \rightarrow A$ and $b: Y \rightarrow B$. If there is a quantum strategy for the players, i.e., if there is a quantum state $\rho \in B\left(H_{A} \otimes H_{B}\right)$ shared by Alice and Bob, and a quantum measurement for Alice (Bob) for each $x \in X(y \in Y)$, for every question $(x, y)$, the probability to output $(a, b)$ is given by $P(a, b \mid x, y)=\operatorname{tr}\left(E_{x}^{a} \otimes F_{y}^{b} \rho\right)$, where $\left(E_{x}^{a}\right)_{x}^{a} \subset B\left(H_{A}\right)$ and $\left(F_{y}^{b}\right)_{y}^{b} \subset B\left(H_{B}\right)$ are POVMs for Alice and $\mathrm{Bob}$, respectively. Thus, the quantum value of the game is

$$
\omega_{q}(G)=\sup \sum_{x, y} \sum_{a, b} \pi(x, y) V(a, b \mid x, y) \operatorname{tr}\left(E_{x}^{a} \otimes F_{y}^{b} \rho\right),
$$

where the sup is taken over all possible quantum strategies.

For a given nonlocal game $G=G(\pi, V)$, there are two natural ways to compare quantum and classical strategies. One is the quotient of the quantum and the classical value, denoted by $\omega_{q}(G) / \omega_{c}(G)$. Another interesting way is to consider the bias of the game. This is the probability of winning the game minus the probability of losing the game. The classical bias $\beta(G)$ [the quantum bias $\beta^{*}(G)$ ] is defined as the maximum bias over all possible classical strategies (quantum strategies). We then consider the quantity $\beta^{*}(G) / \beta(G)$. As we will explain later (see Theorem 2 and Corollary 1), for any nonlocal game with binary answers in one party, one has $\omega_{q}(G) / \omega_{c}(G) \leqslant 2$. Hence, there is no way to get large violations by looking at this quantity. However, we will give an example of these games for which the quantity $\beta^{*}(G) / \beta(G)$ is $\Omega\left[\sqrt{n} /(\log n)^{2}\right]$. To complete the picture, this is equivalent to finding an asymmetric Bell inequality of the form (1) for which the quantity $L V(M)$ is of the order above.

\section{KHOT-VISHNOI GAME AND ITS ASYMMETRIC VERSION}

In this section, we will provide our main result. First, we will recall the KV game and then we will construct an "asymmetric" version of it, which will be a nonlocal game with only two possible answers for Bob's questions. As we will show, the quotient between the quantum bias and the classical bias of the new game can be arbitrarily large, which is equivalent to saying that there is an asymmetric Bell inequality with an unbounded violation.

For any $n=2^{l}$ with $l \in \mathbb{N}$ and $\eta=1 / 2-1 / \log n$, we consider the group of all words in $\{0,1\}^{n}$ and the Hadamard subgroup $H$ with $n$ Hadamard code words. The KV game [21] $G_{\mathrm{KV}}$ is defined as follows: The referee chooses a uniformly random coset $[x] \in\{0,1\}^{n} / H$ and one element $z \in\{0,1\}^{n}$ according to the probability distribution $P\left(z_{i}=\right.$ $1)=\eta, P\left(z_{i}=0\right)=1-\eta$ independently of $i$. Alice and Bob are asked questions $[x]$ and $[x \oplus z]$, respectively, by the 
referee. They answer the outputs $a \in[x]$ and $b \in[x \oplus z]$, and they win the game if and only if $a \oplus b=z$. It is easy to see that the winning probability for a fixed strategy $P$ is

$$
P_{\text {win }}=\frac{n}{2^{n}} \mathbb{E}_{z} \sum_{[x]} \sum_{a \in[x]} P(a, a \oplus z \mid[x],[x \oplus z]) .
$$

Notice that the number of possible inputs to each player is $2^{n} / n$ and the number of possible outputs for each player is $n$. From [21], we know the the violation of this game verifies

$$
\frac{\omega_{q}\left(G_{\mathrm{KV}}\right)}{\omega_{c}\left(G_{\mathrm{KV}}\right)} \geqslant C \frac{n}{(\log n)^{2}},
$$

since $\omega_{c}\left(G_{\mathrm{KV}}\right) \leqslant C_{1} \frac{1}{n}$ and $\omega_{q}\left(G_{\mathrm{KV}}\right) \geqslant C_{2} \frac{1}{(\log n)^{2}}$. Here $C_{1}, C_{2}$, and $C$ are universal constants independent of the dimension. Interestingly, the previous value $\omega_{q}\left(G_{\mathrm{KV}}\right)$ is attained on the maximally entangled state in dimension $n:\left|\psi_{n}\right\rangle=$ $1 / \sqrt{n} \sum_{i=1}^{n}|i i\rangle$. More precisely, the corresponding quantum strategy is given by $\left\langle\psi_{n}\left|E_{[x]}^{a} \otimes E_{[y]}^{b}\right| \psi_{n}\right\rangle$, where for a given $c \in[w], E_{[w]}^{c}=\left|u_{c}\right\rangle\left\langle u_{c}\right|$ for $\left|u_{c}\right\rangle=1 / \sqrt{n} \sum_{i=1}^{n}(-1)^{c(i)}|i\rangle$. In particular, it is known that the previous result is essentially optimal in the number of outputs and in the dimension of the Hilbert spaces (see $[15,25]$ ).

Now we will introduce the asymmetric version of KV game, which plays a paramount role in this paper. Let us start by fixing some notation. Given a coset $[x] \in\{0,1\}^{n} / H$, since $|[x]|=n$ we can identify (by means of a simple enumeration) the coset $[x]$ with the group $\{0,1\}^{l}$. Then, for a given element $a \in[x]$, we will denote by $\tilde{a} \in\{0,1\}^{l}$ its corresponding image. Moreover, for $\tilde{a}, \tilde{b} \in\{0,1\}^{l}$ we denote $\langle\tilde{a}, \tilde{b}\rangle=\sum_{i=1}^{l} \tilde{a}_{i} \tilde{b}_{i}$. With this at hand, we can easily define the asymmetric version of the KV game $G_{\mathrm{KV}}^{\mathrm{as}}$ :

The referee chooses a uniformly random coset $[x] \in$ $\{0,1\}^{n} / H$ and one element $z \in\{0,1\}^{n}$ according to the probability distribution $P\left(z_{i}=1\right)=\eta, P\left(z_{i}=0\right)=1-\eta$ independently of $i$. Moreover, the referee chooses a uniformly random element $k \in\{0,1\}^{l}$. Then, Alice and Bob are asked questions indexed by $[x]$ and $([x \oplus z], k)$, respectively, and they will answer the outputs $a \in[x]$ and $b= \pm 1$. The players win the game if and only if

$$
(-1)^{\langle a \tilde{\oplus} z, k\rangle}=b,
$$

where $a \tilde{\oplus} z \in\{0,1\}^{l}$ is the element associated to $a \oplus z \in[x \oplus z]$.

Theorem 1. If $G_{\mathrm{KV}}^{\mathrm{as}}$ denotes the asymmetric version of the Khot-Visnoi game introduced above, we have

$$
\frac{\beta^{*}\left(G_{\mathrm{KV}}^{\mathrm{as}}\right)}{\beta\left(G_{\mathrm{KV}}^{\mathrm{as}}\right)} \geqslant C \frac{\sqrt{n}}{\log ^{2} n},
$$

where $C$ is an universal constant.

The proof is based on the following estimates:

$$
\beta^{*}\left(G_{\mathrm{KV}}^{\mathrm{as}}\right) \geqslant \omega_{q}\left(G_{\mathrm{KV}}\right) \text { and } \beta\left(G_{\mathrm{KV}}^{\mathrm{as}}\right) \leqslant \sqrt{n} \omega_{c}\left(G_{\mathrm{KV}}\right)
$$

With these two inequalities at hand, we can immediately obtained the statement by invoking Eq. (3) (see Appendix A for details).
Now, for a given strategy $P=(P(a, b \mid[x],([y], k)))_{[x],([y], k)}^{a, b}$, it is straightforward to check that $P_{\text {win }}-P_{\text {lose }}$ is equal to

$$
\frac{1}{2^{n}} \mathbb{E}_{z} \sum_{[x]} \sum_{a \in[x]} \sum_{k \in\{0,1\}^{l}}(-1)^{\langle a \tilde{\oplus} z, k\rangle} E(a \mid[x],([x \oplus z], k)),
$$

where

$$
\begin{aligned}
& E(a \mid[x],([y], k)) \\
& \quad=P(a, 1 \mid[x],([y], k))-P(a,-1 \mid[x],([y], k)) .
\end{aligned}
$$

Let

$$
M_{[x],([y], k)}^{a}=\mathbb{E}_{z} \frac{1}{2^{n}}(-1)^{\langle a \tilde{\oplus} z, k\rangle} \delta_{[y],[x \oplus z]},
$$

where $\delta$ is the Dirac delta function. Then, $M=$ $\left(M_{[x],([y], k)}^{a}\right)_{[x],([y], k)}^{a}$ is an asymmetric Bell inequality in the sense of (1). It is trivial that

$$
\langle M, E\rangle=P_{\text {win }}-P_{\text {lose }} .
$$

Hence, the classical (quantum) bias of the game $G_{\mathrm{KV}}^{\mathrm{as}}$ can be understood as the classical (quantum) bound of the asymmetric Bell inequality $M$. Thus, according to Theorem 1 , we have obtained an asymmetric Bell inequality with a violation of order $\frac{\sqrt{n}}{\log ^{2} n}$.

\section{ASYMMETRIC BELL INEQUALITY WITH NON-NEGATIVE COEFFICIENTS}

The reader could find it surprising that, in order to get large Bell violations in our setting, we have considered the bias of the game $G_{\mathrm{KV}}^{\mathrm{as}}$ in Theorem 1, while in (3) the authors obtained large violations by looking at the values of $G_{\mathrm{KV}}$. The reason is that our context is much more restricted than the case of general answers in two parties, and now we cannot expect the quotient $\omega_{q}\left(G_{\mathrm{KV}}^{\mathrm{as}}\right) / \omega_{c}\left(G_{\mathrm{KV}}^{\mathrm{as}}\right)$ to be large. This can also be understood as the fact that we cannot have large Bell violations of asymmetric Bell inequalities with non-negative entries.

Theorem 2. Let $M$ be a set $\left(M_{x, y}^{a}\right)_{x, y}^{a}$ of non-negative numbers. Then, with the notations in (2),

$$
B_{\mathcal{C}}(M)=B_{\mathcal{Q}}(M) \text {. }
$$

Indeed, note that for any family of POVMs for Alice $\left(E_{x}^{a}\right)_{x}^{a} \subset B\left(H_{A}\right)$ and dichotomic observable for Bob $\left(F_{y}\right)_{y} \subset$ $B\left(H_{B}\right)$, we easily deduce that

$$
\left\|\sum_{x, y, a} M_{x, y}^{a} E_{x}^{a} \otimes F_{y}\right\| \leqslant\left\|\sum_{x, y, a} M_{x, y}^{a} E_{x}^{a}\right\| .
$$

Hence, $B_{\mathcal{Q}}(M)=\left\|\sum_{x, y, a} M_{x, y}^{a} E_{x}^{a} \otimes F_{y}\right\|_{B\left(H_{A} \otimes H_{B}\right)}$ is upper bounded by

$$
\left\|\sum_{x, y, a} M_{x, y}^{a} E_{x}^{a}\right\|_{B\left(H_{A}\right)}=\sup _{\rho}\left|\sum_{x, y, a} M_{x, y}^{a} \operatorname{tr}\left(\rho E_{x}^{a}\right)\right| \leqslant B_{\mathcal{C}}(M) .
$$

Here, the last supremum runs over all states on $H_{A}$ and the last inequality is immediate since $\left[\operatorname{tr}\left(\rho E_{x}^{a}\right)\right]_{x, a}$ is a family of classical probability distributions. This concludes the proof. Note that our asymmetric Bell inequality studied in (6) has signed coefficients.

As a byproduct, for any nonlocal game with binary answers for one player (e.g., for Bob), we have the following result. 
Corollary 1. Let $G=G(\pi, V)$ be a nonlocal game with binary answers for one player. Then,

$$
\omega_{q}(G) \leqslant 2 \omega_{c}(G) \text {. }
$$

Therefore, for such nonlocal games, the ratio of the quantum value and the classical value is bounded by 2 . To obtain a large violation, it is necessary to consider the ratio of the bias.

\section{UPPER BOUNDS FOR THE VIOLATION OF ASYMMETRIC BELL INEQUALITIES}

An interesting property of the KV game is that it offers a near-optimal violation in terms of the number of outputs and the dimension of the Hilbert space. Indeed, for any Bell inequality with $N$ inputs, $K$ outputs, and Hilbert space dimension $d$, we know that the optimal violation is $O(m)$, with $m=\min \{K, N, d\}[15]$. Hence, by (3), the violation of the KV game is near optimal. In this section, we will provide sharp upper bounds for any asymmetric Bell inequality in terms of the output (of Alice) and the Hilbert space dimension. (See Appendix B for the proofs.)

Proposition 1. For any asymmetric Bell inequality $M$, which is defined in (1), the largest violation is upper bounded by $O(\sqrt{K})$.

Proposition 2. For any asymmetric Bell inequality $M$, if the quantum bound of $M$ is achieved by a quantum correlation in which Alice and Bob's local dimension is $d$, then

$$
B_{\mathcal{Q}}(M) \leqslant C \sqrt{d} B_{\mathcal{C}}(M)
$$

where $C$ is a universal constant.

As a corollary of the above propositions, the asymmetric $\mathrm{KV}$ game offers an explicit example of an asymmetric Bell inequality whose violation is essentially optimal (up to a logarithm factor) in the number of Alice's outputs and Hilbert space dimension.

\section{CONCLUSIONS}

In this work, we have provided an example of a bipartite Bell inequality with dichotomic measurements in one party, which can give unbounded Bell violations. This implies a simplification with respect to all previous results where, in order to obtain large Bell violations in the bipartite case, general measurements were needed by both parties. This simplification can be regarded as a new step toward possible experimental realizations. However, reducing the number of measurements seems to be a crucial point since in our example they scale exponentially. On the other hand, our result is essentially optimal in Alice's outputs and in the Hilbert space dimension. Additionally, the asymmetry considered in this work can also be interesting for device-independent scenarios, where it seems very reasonable to assume that Alice and Bob have different kinds of measurements. Finally, from a computer science point of view our result can be considered as an example of a nonlocal game, for which the quotient of the quantum bias and the classical bias can be arbitrarily large. Interestingly, we do need to consider the bias of the game since, in contrast with more general settings, in our context we cannot use the classical and the quantum value of the game to obtain large violations.

\section{ACKNOWLEDGMENTS}

We would like to thank David Pérez-García, Michał Horodecki, Marcin Marciniak, and Marius Junge for many helpful discussions. C.P. was supported by Spanish research projects MTM2011-26912, PRI-PIMCHI-2011-1071 (part of CHIST-ERA CQC), QUITEMAD+-CM, ref. S2013/ICE2801, and the "Ramón y Cajal" program. Z.Y. acknowledge support by the TEAM project of FNP, ERC Grant QOLAPS, EC Grant RAQUEL, and NSFC under Grant No. 11301401.

\section{APPENDIX A}

The proof of Theorem 1 is based on the following estimates:

$$
\beta^{*}\left(G_{\mathrm{KV}}^{\mathrm{as}}\right) \geqslant \omega_{q}\left(G_{\mathrm{KV}}\right) \text { and } \beta\left(G_{\mathrm{KV}}^{\mathrm{as}}\right) \leqslant \sqrt{n} \omega_{c}\left(G_{\mathrm{KV}}\right)
$$

To see the first inequality in (5), let us assume that Alice and Bob have a quantum strategy for the KV game defined by an entangled state $|\psi\rangle$ and two families of POVMs $\left(E_{[x]}^{a}\right)_{[x]}^{a}$, $\left(F_{[y]}^{b}\right)_{[y]}^{b}$. Then, Alice and Bob can define another strategy for the asymmetric version of the $\mathrm{KV}$ game consisting of sharing the same quantum state, and, moreover, Alice's strategy is also defined by the family $\left(E_{[x]}^{a}\right)_{[x]}^{a}$. On the other hand, for a given question $([y], k)$ to Bob, he will consider the self-adjoint operator

$$
B_{[y], k}=\sum_{b \in[y]}(-1)^{\langle\tilde{b}, k\rangle} F_{[y]}^{b} .
$$

It is clear that $-\mathbb{1} \leqslant B_{[y], k} \leqslant \mathbb{1}$ for every $([y], k)$. Hence, we conclude that $\beta^{*}\left(G_{\mathrm{KV}}^{\mathrm{as}}\right)$ is lower bounded by

$$
\begin{aligned}
& \frac{1}{2^{n}} \mathbb{E}_{z} \sum_{[x]} \sum_{a \in[x]} \sum_{k \in\{0,1\}^{l}}(-1)^{\langle a \tilde{\oplus} z, k\rangle}\left\langle\psi\left|E_{[x]}^{a} \otimes B_{[x \otimes z], k}\right| \psi\right\rangle
\end{aligned}
$$

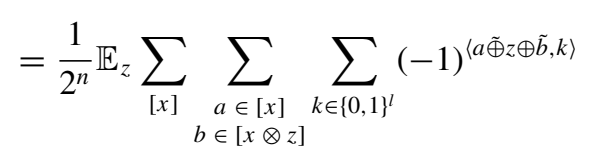

$$
\begin{aligned}
& \times\left\langle\psi\left|E_{[x]}^{a} \otimes F_{[x \otimes z]}^{b}\right| \psi\right\rangle \\
& =\frac{n}{2^{n}} \mathbb{E}_{z} \sum_{[x]} \sum_{a \in[x]}\left\langle\psi\left|E_{[x]}^{a} \otimes F_{[x \otimes z]}^{a \oplus z}\right| \psi\right\rangle .
\end{aligned}
$$

That is, for every strategy performed by Alice and Bob for the $\mathrm{KV}$ game, we can define another strategy for the asymmetric version of the $\mathrm{KV}$ such that the quantum bias for the second one is lower bounded by the quantum value for the first one.

To show the second inequality in (5), let us fix a classical strategy (correlation) for the $G_{\mathrm{KV}}^{\mathrm{as}}$. It suffices to look at the extreme points, so we can assume that $E(a \mid[x],([y], k))=$ $P(a \mid[x]) E([y], k)$ with $P(a \mid[x]) \geqslant 0$ and $\sum_{a} P(a \mid x)=1$ for every $x, a$ and $-1 \leqslant E([y], k) \leqslant 1$ for every $([y], k)$. Then, we define

$$
Q(b \mid[y])=\sum_{k \in\{0,1\}^{l}}(-1)^{\langle\tilde{b}, k\rangle} E([y], k) \text { for every }[y], b \in[y] .
$$

We claim that

$$
n^{-\frac{3}{2}} \sum_{b \in[y]}|Q(b \mid[y])| \leqslant 1 \text { for every }[y] .
$$


Then, if we consider $P=n^{-\frac{3}{2}}(P(a \mid[x]) Q(b \mid[y]))_{[x],[y], a, b}$, we can easily deduce that $\left\langle G_{\mathrm{KV}}, P\right\rangle$ equals

$$
\frac{1}{\sqrt{n} 2^{n}} \mathbb{E}_{z} \sum_{[x]} \sum_{a \in[x]} \sum_{k \in\{0,1\}^{l}}(-1)^{\langle a \tilde{\oplus} z, k\rangle} P(a \mid[x]) E([x \oplus z], k) .
$$

On the other hand, the fact that the KV game has positive coefficients (as a Bell inequality) guarantees that $\omega_{c}\left(G_{\mathrm{KV}}\right) \geqslant$ $\left\langle G_{\mathrm{KV}}, P\right\rangle$, since we could always improve the previous value by modifying the $n^{-\frac{3}{2}} Q(b \mid[y])$ 's so that all they are positive and they sum up to 1 .

Since the last expression above is the same as the classical bias of the asymmetric KV game when we consider the correlation $(E(a \mid[x],([y], k)))_{[x],([y], k)}^{a}$, we deduce our result.

Finally, in order to show our claim (A2), we note that $\sum_{b \in[y]}|Q(b \mid[y])|$ is equal to

$$
\begin{aligned}
& \sum_{b \in[y]}\left|\sum_{k \in\{0,1\}^{l}}(-1)^{\langle\tilde{b}, k\rangle} E([y], k)\right| \\
& \leqslant \sqrt{n}\left(\sum_{b \in[y]}\left|\sum_{k \in\{0,1\}^{l}}(-1)^{\langle\tilde{b}, k\rangle} E([y], k)\right|^{2}\right)^{\frac{1}{2}} \\
& =\sqrt{n}\left(\sum_{b \in[y]} \sum_{k, k^{\prime} \in\{0,1\}^{l}}(-1)^{\left\langle\tilde{b}, k \oplus k^{\prime}\right\rangle} E([y], k) E\left([y], k^{\prime}\right)\right)^{\frac{1}{2}} \\
& =n\left(\sum_{k \in\{0,1\}^{l}} E([y], k)^{2}\right)^{\frac{1}{2}} \leqslant n^{\frac{3}{2}} \sup _{k \in\{0,1\}^{l}}|E([y], k)| \leqslant n^{\frac{3}{2}} .
\end{aligned}
$$

Hence we complete the proof of Theorem 1.

For the proof of Corollary 1, we note that

$$
\begin{aligned}
\omega_{q}(G) & =\sup \left\|\sum_{x, y, a, b} \pi(x, y) V(a, b \mid x, y) E_{x}^{a} \otimes F_{y}^{b}\right\|_{B\left(H_{A} \otimes H_{B}\right)} \\
& \leqslant \sup \left\|\sum_{x, y, a, b} \pi(x, y) V(a, b \mid x, y) E_{x}^{a} \otimes A_{(y, b)}\right\| \|_{B\left(H_{A} \otimes H_{B}\right)} \\
& =\sup \left|\sum_{x, y, a, b} \pi(x, y) V(a, b \mid x, y) P(a \mid x) \alpha_{(y, b)}\right| .
\end{aligned}
$$

Here, the first supremum runs over all pairs of POVMs $\left(E_{x}^{a}\right)_{x}^{a},\left(F_{y}^{b}\right)_{y}^{b}$ for Alice and Bob, respectively, while in the second one $-11 \leqslant A_{(y, b)} \leqslant \mathbb{1}$ for every $(y, b)$. The last equality follows Theorem 2, where now the last supremum runs over all classical strategies $[P(a \mid x)]_{x}^{a}$ and $-1 \leqslant$ $\alpha_{(y, b)} \leqslant 1$. Note that in order to use the theorem, we must view $M=\left(M_{x,(y, b)}^{a}\right)_{x,(y, b)}^{a}$ as an asymmetric Bell inequality, where $M_{x,(y, b)}^{a}=\pi(x, y) V(a, b \mid x, y)$. Finally, it is very easy to see that the last quantity is upper bounded by $2 \sup \left|\sum_{x, y, a, b} \pi(x, y) V(a, b \mid x, y) P(a \mid x) \alpha_{y}^{b}\right|$, where this supremum runs over all families $\left(\alpha_{y}^{b}\right)_{y}^{b}$ such that $\left|\alpha_{y}^{0}\right|+$ $\left|\alpha_{y}^{1}\right| \leqslant 1$ for every $y$. Using the fact that the numbers $\pi(x, y) V(a, b \mid x, y) P(a \mid x)$ are non-negative, one can easily conclude that the last supremum is upper bounded by $\omega_{c}(G)$, and the proof follows.

\section{APPENDIX B}

The proof of Proposition 1 is based on Grothendieck's inequality for complex matrices, and it follows the same ideas as in Sec. 5 in Ref. [15]. Given a complex matrix $\left(M_{x, y}\right)_{x, y}$ with $x=1, \ldots, N, y=1, \ldots, N^{\prime}$, let us denote

$$
\begin{aligned}
& B_{\mathcal{C}}(M)=\sup \left\{\left|\sum_{x, y} M_{x, y} t_{x} s_{y}\right|\right\} \text { and } \\
& B_{\mathcal{Q}}(M)=\sup \left\{\left|\sum_{x, y} M_{x, y}\left\langle u_{x}, v_{y}\right\rangle\right|\right\},
\end{aligned}
$$

where the first supremum runs over all complex numbers $t_{x}$, $s_{y}$ with $\left|t_{x}\right|,\left|s_{y}\right| \leqslant 1$ for all $x, y$ and the second supremum runs over all complex Hilbert spaces $H$ and vectors $u_{x}, v_{y}$ in the unit ball of $H$ for all $x, y$. Grothendieck's inequality (see, for instance, Chap. 14 in Ref. [26]) states that there exists a positive constant $C$ such that for any complex matrix $\left(M_{x, y}\right)_{x, y}$

$$
B_{\mathcal{Q}}(M) \leqslant C B_{\mathcal{C}}(M) .
$$

The smallest constant verifying inequality (B1) is called (complex) Grothendieck's constant $K_{G}^{\mathbb{C}}$, and, although its exact value is still unknown, it verifies $1.338 \leqslant K_{G}^{\mathbb{C}} \leqslant 1.405$.

For a family of complex numbers $E=[E(x, y)]_{x, y}$, we will denote

$$
\langle M, E\rangle=\sum_{x=1}^{N} \sum_{y=1}^{N^{\prime}} M_{x, y} E(x, y)
$$

For the proof of the proposition, it suffices to consider the case $N=N^{\prime}$, i.e., Alice and Bob have the same number of inputs. Let us consider an arbitrary asymmetric Bell inequality $M=\left(M_{x, y}^{a}\right)_{x, y}^{a}$ with $x, y=1, \ldots, N$ and $a=1, \ldots, K$. Then, we define the following $(N+1)(K-1) \times N$ complex matrix:

$$
\begin{aligned}
& M_{(x, s), y}^{\prime}=\sum_{a=1}^{K-1} \omega^{a s}\left(M_{x, y}^{a}-M_{x, y}^{K}\right), \begin{array}{l}
x, y=1, \ldots, N \\
s=1, \ldots, K-1, \\
M_{(N+1, s), y}^{\prime}=\sum_{x=1}^{N} \omega^{s} M_{x, y}^{K},
\end{array} \\
& \begin{array}{l}
y=1, \ldots, N \\
s=1, \ldots, K-1
\end{array},
\end{aligned}
$$

where $\omega=\exp \frac{2 \pi i}{K-1}$. According to (B1) we have that

$$
B_{\mathcal{Q}}\left(M^{\prime}\right) \leqslant K_{G}^{\mathbb{C}} B_{\mathcal{C}}\left(M^{\prime}\right) .
$$

Our upper bound will follow from the estimates

$$
\begin{aligned}
& B_{\mathcal{C}}\left(M^{\prime}\right) \leqslant 24(K-1)^{\frac{3}{2}} B_{\mathcal{C}}(M) \\
\text { and } \quad & B_{\mathcal{Q}}\left(M^{\prime}\right) \geqslant(K-1) B_{\mathcal{Q}}(M) .
\end{aligned}
$$

Let us start proving the second inequality. Toward that end, let us consider an arbitrary quantum strategy $\left[\operatorname{tr}\left(\rho E_{x}^{a} \otimes\right.\right.$ $\left.\left.B_{y}\right)\right]_{x, y=1 ; a=1}^{N ; K}$ for $M$. In fact, by convexity we can assume that $\rho$ is a pure state, so that our strategy is of the form $\left(\left\langle\psi\left|E_{x}^{a} \otimes B_{y}\right| \psi\right\rangle\right)_{x, y=1 ; a=1}^{N ; K}$, where $|\psi\rangle$ is a unit vector. Then, 
we define the following family of operators:

$$
\begin{array}{ll}
A_{(x, s)}=\sum_{a=1}^{K-1} \omega^{-a s} E_{x}^{a}, & \begin{array}{l}
x=1, \ldots, N \\
s=1, \ldots, K-1
\end{array} \\
A_{(N+1, s)}=\omega^{-s} \mathbb{1 1}, & s=1, \ldots, K-1 .
\end{array}
$$

It is straightforward to check that $\left\|A_{(x, s)}\right\| \leqslant 1$ for every $(x, s)$. Therefore, $u_{x, s}=\left(A_{(x, s)} \otimes \mathbb{1 1}\right)|\psi\rangle$ and $v_{y}=(\mathbb{1} \otimes$ $\left.B_{y}\right)|\psi\rangle$ form a family of vectors in the unit Ball of a complex Hilbert space such that $\gamma=\left(\left\langle u_{x, s}, v_{y}\right\rangle\right)_{(x, s), y}=\left(\langle\psi| A_{(x, s)} \otimes\right.$ $\left.B_{y}|\psi\rangle\right)_{(x, s), y}$. Thus, $B_{\mathcal{Q}}\left(M^{\prime}\right)$ is lower bounded by

$$
\begin{aligned}
\left|\left\langle M^{\prime}, \gamma\right\rangle\right|= & \left|\sum_{x=1}^{N+1} \sum_{y=1}^{N} \sum_{s=1}^{K-1} M_{(x, s), y}^{\prime}\left\langle\psi\left|A_{(x, s)} \otimes B_{y}\right| \psi\right\rangle\right| \\
= & \mid \sum_{x, y=1}^{N} \sum_{s, a, a^{\prime}} \omega^{\left(a-a^{\prime}\right) s}\left(M_{x, y}^{a}-M_{x, y}^{K}\right)\left\langle\psi\left|E_{x}^{a^{\prime}} \otimes E_{y}\right| \psi\right\rangle \\
& +\sum_{x, y=1}^{N} \sum_{s=1}^{K-1} M_{x, y}^{K}\left\langle\psi\left|11 \otimes E_{y}\right| \psi\right\rangle \mid \\
= & (K-1)\left|\sum_{x, y=1}^{N} \sum_{a=1}^{K} M_{x, y}^{a}\left\langle\psi\left|E_{x}^{a} \otimes E_{y}\right| \psi\right\rangle\right| .
\end{aligned}
$$

For the last equality, we have used that $\sum_{a=1}^{K-1} E_{x}^{a}=\mathbb{1}-E_{x}^{K}$ for every $x=1, \ldots, N$. This proves the second estimate in (B2).

To see the first estimate in (B2), let us consider an element $\left[R(x, s) \beta_{y}\right]_{x, s, y}$ such that $|R(x, s)| \leqslant 1$ and $\left|\beta_{y}\right| \leqslant 1$ for all $x=1, \ldots, N+1, s=1, \ldots, K-1, y=1, \ldots, N$. Then, we define the following object $P$ : For every $x=1, \ldots, N$,

$$
\begin{array}{r}
P(a \mid x)=\sum_{s=1}^{K-1} \omega^{a s} R(x, s), \quad a=1, \ldots, K-1, \\
P(K \mid x)=\sum_{s=1}^{K-1} \omega^{s} R(N+1, s)-\sum_{a, s=1}^{K-1} \omega^{a s} R(x, s) .
\end{array}
$$

Note that, for fixed $x=1, \ldots, N$,

$$
\begin{aligned}
\sum_{a=1}^{K} P(a \mid x)= & \sum_{a, s=1}^{K-1} \omega^{a s} R(x, s)+\sum_{s=1}^{K-1} \omega^{s} R(N+1, s) \\
& -\sum_{a, s=1}^{K-1} \omega^{a s} R(x, s)=\sum_{s=1}^{K-1} \omega^{s} R(N+1, s),
\end{aligned}
$$

which is a constant independent of $x$.

On the other hand,

$$
\begin{aligned}
\sum_{a=1}^{K}|P(a \mid x)| \leqslant & 2 \sum_{a=1}^{K-1}\left|\sum_{s=1}^{K-1} \omega^{a s} R(x, s)\right| \\
& +(K-1) \leqslant 3(K-1)^{3 / 2},
\end{aligned}
$$

where the last inequality is proved exactly in a similar way as claim (A2) in Theorem 1.

The previous two properties together with Lemma 3.2 in Ref. [25] guarantee the existence of two classical strategies $P_{1}$ and $P_{2}$ for Alice and $\lambda_{1}, \lambda_{2} \in \mathbb{R}$ such that $\operatorname{Re}(P)=\lambda_{1} P_{1}+$ $\lambda_{2} P_{2}$ and $\left|\lambda_{1}\right|+\left|\lambda_{2}\right| \leqslant 3(K-1)^{3 / 2}$. Here, $\operatorname{Re}(P)$ denotes the real part of $P$. The same argument holds for the imaginary part $\operatorname{Im}(P)$, i.e., there exist classical strategies $P_{3}$ and $P_{4}$ for Bob and $\lambda_{3}, \lambda_{4} \in \mathbb{R}$ such that $\operatorname{Im}(P)=\lambda_{3} P_{3}+\lambda_{4} P_{4}$ and $\left|\lambda_{3}\right|+$ $\left|\lambda_{4}\right| \leqslant 3(K-1)^{3 / 2}$. On the other hand, for every $y$ we can write $\beta_{y}=\beta_{y}^{1}+i \beta_{y}^{2}$ with $\beta_{y}^{1}, \beta_{y}^{2}$ real numbers verifying $\left|\beta_{y}^{j}\right| \leqslant$ 1 for $j=1,2$. Hence, our estimate will follow from the fact that $24(K-1)^{3 / 2} B_{\mathcal{C}}(M)$ is lower bounded by

$$
\begin{aligned}
3(K & -1)^{3 / 2} \sum_{i=1}^{4} \sum_{j=1}^{2} \mid\left\langle M,\left[P_{i}(a \mid x) \beta_{y}^{j}\right]_{x, y, a}||\right. \\
\geqslant & \left|\left\langle M,\left[P(a \mid x) \beta_{y}\right]_{x, y, a}\right\rangle\right|=\mid \sum_{x, y=1}^{N} \sum_{a, s=1}^{K-1} M_{x, y}^{a} \omega^{a s} R(x, s) \beta_{y} \\
& +\sum_{x, y=1}^{N} M_{x, y}^{K}\left[\sum_{s=1}^{K-1} \omega^{s} R(N+1, s)-\sum_{a, s=1}^{K-1} \omega^{a s} R(x, s)\right] \beta_{y} \mid \\
= & \mid \sum_{x, y=1}^{N} \sum_{a, s=1}^{K-1}\left(M_{x, y}^{a}-M_{x, y}^{K}\right) \omega^{a s} R(x, s) \beta_{y} \\
& +\sum_{x, y=1}^{N} M_{x, y}^{K} \sum_{s=1}^{K-1} \omega^{s} R(N+1, s) \beta_{y} \mid \\
= & \left|\left\langle M^{\prime},\left[R(x, s) \beta_{y}\right]_{x, s, y=1}^{N+1, K-1, N}\right\rangle\right| .
\end{aligned}
$$

By taking the supremum over all elements $\left[R(x, s) \beta_{y}\right]_{x, s, y}$, we prove the first inequality in (B2).

The result of Proposition 2 can be proven by following the same ideas as in Theorem 3 in Ref. [20]. Here, we will provide a sketch of proof. Given $\epsilon>0$, let $|\Psi\rangle,\left(E_{x}^{a}\right)_{x, a},\left(A_{y}\right)_{y}$ be a quantum state, some POVMs for Alice and dichotomic observables for Bob, respectively, such that

$$
B_{\mathcal{Q}}(M) \leqslant(1+\epsilon)\left|\sum_{x, y, a} M_{x, y}^{a}\left\langle\Psi\left|E_{x}^{a} \otimes A_{y}\right| \Psi\right\rangle\right| .
$$

Note that $A_{y} \in B\left(H_{d}\right)$ for every $y$. Let $|\Psi\rangle=\sum_{i=1}^{d} \lambda_{i}\left|u_{i}\right\rangle\left|v_{i}\right\rangle$ be the Schmidt decomposition, where $\left(\left|v_{i}\right\rangle\right)_{i}$ span the local Hilbert space of Bob. For every $y$, let $M_{y}=$ $\sum_{x, a} M_{x, y}^{a} E_{x}^{a}$ and $E_{i, j}=\sum_{y}\left\langle v_{i}\left|A_{y}\right| v_{j}\right\rangle M_{y}$. Then, it is clear that $M=\sum_{i, j} E_{i, j} \otimes\left|v_{i}\right\rangle\left\langle v_{j}\right|$ verifies that $|\langle\Psi|M| \Psi\rangle| \geqslant(1+$ $\epsilon)^{-1} B_{\mathcal{Q}}(M)$. On the other hand, since $M$ is Hermitian we easily deduce that $E_{i, j}=E_{j, i}^{\dagger}$ for every $i, j$. Therefore, by the same argument as in Claim 7 in Ref. [20], for every $i$ we have

$$
\max \left\{\left\|\sum_{j} E_{i, j} E_{i, j}^{\dagger}\right\|,\left\|\sum_{j} E_{i, j}^{\dagger} E_{i, j}\right\|\right\} \leqslant 4 K_{G}^{\mathbb{C}} B_{\mathcal{C}}(M)^{2} .
$$

The universal constant is slightly different from the one appearing in Claim 7 in Ref. [20], since here we consider a bipartite case. Now, by the noncommutative Khinchine inequality (Theorem 6 in Ref. [20]), one can show

$$
|\langle\Psi|M| \Psi\rangle| \leqslant 6\left(K_{G}^{\mathbb{C}}\right)^{\frac{1}{2}} \sqrt{d} B_{\mathcal{C}}(M) .
$$

Since the argument is the same as that in Theorem 3 of Ref. [20], we omit the details. 
[1] A. Einstein, B. Podolsky, and N. Rosen, Phys. Rev. 47, 777 (1935).

[2] J. S. Bell, Physics (N.Y.) 1, 195 (1964).

[3] N. Brunner, D. Cavalcanti, S. Pironio, V. Scarani, and S. Wehner, Rev. Mod. Phys. 86, 419 (2014).

[4] N. Brunner, S. Pironio, A. Acín, N. Gisin, A. A. Méthot, and V. Scarani, Phys. Rev. Lett. 100, 210503 (2008).

[5] P. Hyllus, O. Gühne, D. Bruß, and M. Lewenstein, Phys. Rev. A 72, 012321 (2005).

[6] B. M. Terhal, Phys. Lett. A 271, 319 (2000).

[7] C. Brukner, M. Zukowski, J. W. Pan, and A. Zeilinger, Phys. Rev. Lett. 92, 127901 (2004).

[8] A. Acín, N. Brunner, N. Gisin, S. Massar, S. Pironio, and V. Scarani, Phys. Rev. Lett. 98, 230501 (2007).

[9] A. Acín, N. Gisin, and L. Masanes, Phys. Rev. Lett. 97, 120405 (2006).

[10] U. Vazirani and T. Vidick, Phys. Rev. Lett. 113, 140501 (2014).

[11] S. Pironio, A. Acín, S. Massar, A. Boyer de la Giroday, D. N. Matsukevich, P. Maunz, S. Olmschenk, D. Hayes, L. Luo, T. A. Manning, and C. Monroe, Nature (London) 464, 1021 (2010).

[12] U. Vazirani and T. Vidick, Philos. Trans. R. Soc. London, Ser. A 370, 3432 (2012).

[13] H. Buhrman, L. Czekaj, A. Grudka, M. Horodecki, P. Horodecki, M. Markiewicz, F. Speelman, and S. Strelchuk, arXiv:1502.01058.
[14] D. Cavalcanti, R. Rabelo, and V. Scarani, Phys. Rev. Lett. 108, 040402 (2012).

[15] M. Junge and C. Palazuelos, Commun. Math. Phys. 306, 695 (2011).

[16] C. Palazuelos, Phys. Rev. Lett. 109, 190401 (2012).

[17] B. Tsirelson, Hadronic J. 8, 329 (1993).

[18] D. Pérez-García, M. M. Wolf, C. Palazuelos, I. Villanueva, and M. Junge, Commun. Math. Phys. 279, 455 (2008).

[19] M. Junge, C. Palazuelos, D. Pérez-García, I. Villanueva, and M. M. Wolf, Commun. Math. Phys. 300, 715 (2010).

[20] J. Briet and T. Vidick, Commun. Math. Phys. 321, 181 (2013).

[21] H. Buhrman, O. Regev, G. Scarpa, and R. de Wolf, IEEE 26th Annual Conference on Computational Complexity (IEEE, Piscataway, NJ, 2011), pp. 157-166.

[22] O. Regev, Quantum Inf. Comput. 12, 9 (2012).

[23] S. Khot and N.Vishnoi, in Proceedings of 46th IEEE Symposium on Foundations of Computer Science (FOCS), Pittsburgh, (2005) (IEEE, Piscataway, NJ, 2005), pp. 53-62.

[24] R. Cleve, P. Høyer, B. Toner, and J. Watrous, IEEE 19th Annual Conference on Computational Complexity (IEEE, Piscataway, NJ, 2004), pp. 236-249.

[25] C. Palazuelos, J. Funct. Anal. 267, 1959 (2014).

[26] A. Defant and K. Floret, Tensor Norms and Operator Ideals, North-Holland Math, Studies North-Holland, Amsterdam (1993). 